\title{
Phase Measurement in Interacting Fock Space
}

\author{
Luigi Accardi ${ }^{1,3}$ and P. K. Das ${ }^{2}$
}

Received May 15, 2003

In this paper we discuss probability operator measure and phase measurement in one mode interacting Fock space.

KEY WORDS: phase measurement; Fock space; POM.

\section{INTRODUCTION}

Refractive index variations because of various environmental effects cause phase shifts to optical beams which we can observe interferometrically. In a coherent state (shot-noise limited) phase sensing interferometer, the root-mean-square phase error is proportional to $1 / \sqrt{N}$, where $N$ is the average number of detected photons. If we use squeezed states in a single-frequency Michelson interferometer gravity-wave detector then root-mean-square phase error is proportional to $1 / N$. This means that a squeezed state interferometer requires fewer photons than does a conventional coherent-state interferometer to reach the standard quantum limit on position measurement accuracy. But squeezed state interferometry does not represent the ultimate quantum limit on the measurement of optical phase. Better performance can be achieved in phase measurement problems by using quantum estimation theory. As the statistics of a quantum measurement depends on both the measurement operator and the input state, one studies the phase measurement problem on a single-mode radiation field, optimizing both the quantum state and the quantum measurement, and arrives at a local accuracy $\delta \phi \sim 1 / N^{2}$ which is the reciprocal peak likelihood error and not the root-mean-square error.

In this direction Shapiro and others studied phase measurements on a singlemode radiation field of the annihilation operator $a$ on a Boson-Fock space. They

${ }^{1}$ Centro Matematico Vito Volterra, Università di Roma “Tor Vergata," Facoltà di Economia, Via di Tor Vergata snc, Roma, Italy.

${ }^{2}$ Physics and Applied Mathematics Unit, Indian Statistical Institute, Kolkata, India.

${ }^{3}$ To whom correspondence should be addressed at Centro Matematico Vito Volterra, Università di Roma "Tor Vergata," Facoltà di Economia, Via di Tor Vergata snc, 00133 Roma, Italy; e-mail: accardi@volterra.mat.uniromaz.it. 
further studied probability operator measures (POM) and showed that the POM generated by the Susskind-Glogower operator yields the maximum-likelihood phase estimation for an arbitrary input state.

Taking the view point of Shapiro and others we study here the same problem in the interacting Fock space setting and observe that their result can be improved further in the new setting.

The paper is organized as follows. In Section 2, we give preliminaries and notations. In Section 3, we describe probability operator measure in $\Gamma(\mathbb{C})$. In Section 4, we discuss phase distribution in $\Gamma(\mathbb{C})$. In Section 5, we study phase estimation problem and in Section 6 we have given a conclusion.

\section{PRELIMINARIES AND NOTATIONS}

As a vector space one mode interacting Fock space $\Gamma(\mathbb{C})$ is defined by

$$
\Gamma(\mathbb{C})=\bigoplus_{n=0}^{\infty} \mathbb{C}|n\rangle .
$$

where $\mathbb{C}|n\rangle$ is called the $n$-particle subspace. The different $n$-particle subspaces are orthogonal, that is, the sum in (1) is orthogonal. The norm of the vector $|n\rangle$ is given by

$$
\langle n \mid n\rangle=\lambda_{n} .
$$

where $\left\{\lambda_{n}\right\}>0$. The norm introduced in (2) makes $\Gamma(\mathbb{C})$ a Hilbert space.

An arbitrary vector $f$ in $\Gamma(\mathbb{C})$ is given by

$$
f \equiv r_{0}|0\rangle+c_{1}|1\rangle+c_{2}|2\rangle+\cdots+c_{n}|n\rangle+\cdots
$$

with $\|f\|=\left(\sum_{n=0}^{\infty}\left|c_{n}\right|^{2} \lambda_{n}\right)^{1 / 2}<\infty$.

We now consider the following actions on $\Gamma(\mathbb{C})$ :

$$
\begin{aligned}
a^{+}|n\rangle & =|n+1\rangle \\
a|n+1\rangle & =\frac{\lambda_{n+1}}{\lambda_{n}}|n\rangle
\end{aligned}
$$

$a^{+}$is called the creation operator and its adjoint $a$ is called the annihilation operator. To define the annihilation operator we have taken the convention $0 / 0=0$.

We observe that

$$
\langle n \mid n\rangle=\left\langle a^{+}(n-1), n\right\rangle=\langle(n-1), a n\rangle=\frac{\lambda_{n}}{\lambda_{n-1}}\langle n-1, n-1\rangle=\ldots
$$

and

$$
\| n\rangle \|^{2}=\frac{\lambda_{n}}{\lambda_{n-1}} \cdot \frac{\lambda_{n-1}}{\lambda_{n-2}} \cdots \frac{\lambda_{1}}{\lambda_{0}}=\frac{\lambda_{n}}{\lambda_{0}}
$$


By (2) we observe from (6) that $\lambda_{0}=1$.

The commutation relation takes the form

$$
\left[a, a^{+}\right]=\frac{\lambda_{N+1}}{\lambda_{N}}-\frac{\lambda_{N}}{\lambda_{N-1}}
$$

where $N$ is the number operator defined by $N|n\rangle=n|n\rangle$.

In a recent paper (Das, 2002), we have proved that the set $\left\{f_{n} \equiv \frac{|n\rangle}{\sqrt{\lambda_{n}}}, n=\right.$ $0,1,2,3, \ldots\}$ forms a complete orthonormal set.

We have shown (Das, 2002) that the solution of the following eigenvalue equation

$$
a f_{\alpha}=\alpha f_{\alpha}
$$

is given by

$$
f_{\alpha}=\psi\left(|\alpha|^{2}\right)^{-1 / 2} \sum_{n=0}^{\infty} \frac{\alpha^{n}}{\lambda_{n}}|n\rangle
$$

where $\psi\left(|\alpha|^{2}\right) \equiv \sum_{n=0}^{\infty} \frac{|\alpha|^{2 n}}{\lambda_{n}}$.

We call $f_{\alpha}$ a coherent vector in $\Gamma(\mathbb{C})$.

\section{PROBABILITY OPERATOR MEASURE}

A discrete spectrum Probability Operator Measure (POM) on $\Gamma(\mathbb{C})$ consists of a set of Hermitian, positive semidefinite operators $\left\{\Pi_{n}: n \in \mathbb{N}\right\}$, which resolves the identity

$$
I=\sum_{n \in \mathbb{N}} \Pi_{n}
$$

Measurement of this POM, by definition, gives a discrete, classical random variable with probability distribution

$$
P(n, g)=\left(g, \Pi_{n} g\right) \text { for } n \in \mathbb{N}
$$

where $g$ is any vector of unit norm in $\Gamma(\mathbb{C})$.

In order that the laws of classical probability be satisfied, it is necessary and sufficient that

$$
0 \leq P(n, g) \leq 1, \quad \sum_{n=0}^{\infty} P(n, g)=1
$$

are satisfied for arbitrary $g$ of unit norm in $\Gamma(\mathbb{C})$.

We know that the sequence $f_{n}=\frac{|n\rangle}{\lambda_{n}}$ forms a complete orthonormal sequence in $\Gamma(\mathbb{C})$ and are eigenvectors of the operator $N^{\prime}=a^{*} a$ such that

$$
N^{\prime} f_{n}=\frac{\lambda_{n}}{\lambda_{n-1}} f_{n} \text {. }
$$


Measurement of $N^{\prime}$ for any arbitrary vector $g \in \Gamma(\mathbb{C})$ of unit norm yields a discrete-valued, classical random variable with probability distribution

$$
P\left(f_{n}, g\right)=\left|\left(f_{n}, g\right)\right|^{2} \quad \text { for } n=0,1,2, \ldots
$$

In order that the law of classical probability be satisfied it is necessary and sufficient that

$$
0 \leq P\left(f_{n}, g\right) \leq 1, \quad \sum_{n=0}^{\infty} P\left(f_{n}, g\right)=1
$$

for arbitrary $g \in \Gamma(\mathbb{C})$ of unit norm.

The completeness of $\left\{f_{n}\right\}$ guarantees that the prescription in Eq. (14) obeys Eq. (15). For, if we expand the arbitrary vector $g$ of unit norm in terms of $f_{n}$ we have

$$
g=\sum_{n=0}^{\infty}\left(f_{n}, g\right) f_{n}=\sum_{n=0}^{\infty}\left|f_{n}\right\rangle\left\langle f_{n}\right| g
$$

where we define the operator

$$
\left|f_{n}\right\rangle\left\langle f_{n}\right|: \Gamma(\mathbb{C}) \rightarrow \Gamma(\mathbb{C})
$$

by

$$
\left|f_{n}\right\rangle\left\langle f_{n}\right|=\left(f_{n}, g\right) f_{n} .
$$

Equation (15) is now easily verified from Eqs. (14) (16).

Thus, $N^{\prime}$ operator measurement is equivalent to the POM

$$
\left\{\Pi_{n}=\left|f_{n}\right\rangle\left\langle f_{n}\right|: n=0,1,2, \ldots\right\} .
$$

Similarly, a continuous spectrum POM consists of a set of Hermitian, positive semidefinite differential operators $\{d \Pi(\beta): \beta \in \mathbb{C}\}$, which resolve the identity,

$$
I=\int_{\beta \in \mathscr{C}} d \Pi(\beta) .
$$

The result of measuring this POM is, by definition, a continuous, classical random variable whose probability density function is given by

$$
p(\beta, g)=\frac{(g, d \Pi(\beta) g)}{d \beta} \text { for } \beta \in \mathbb{C}
$$

where $g$ is any vector of unit norm in $\Gamma(\mathbb{C})$.

We know that the annihilation operator $a$ has eigenvectors - the coherent vectors $f_{\alpha}$ (Das, 2002). These vectors are not orthogonal but they form a resolution of the identity

$$
I=\int_{\alpha \in \mathscr{C}^{\prime}} d \mu(\alpha)\left|f_{\alpha}\right\rangle\left\langle f_{\alpha}\right|
$$

where

$$
d \mu(\alpha)=\psi\left(|\alpha|^{2}\right) \sigma\left(|\alpha|^{2}\right) r d r d \theta
$$


with $\alpha=r e^{i \theta}$, which defines a $a$-POM

$$
d \Pi(\alpha) \equiv\left|f_{\alpha}\right\rangle\left\langle f_{\alpha}\right| d \mu(\alpha) \text { for } \quad \alpha \in \mathbb{C} .
$$

The outcome of the $a$-POM is a complex-valued, continuous classical random variable with probability density function

$$
p(\alpha, g)=\frac{(g, d \Pi(\alpha) g)}{d \mu(\alpha)}=\left|\left(f_{\alpha}, g\right)\right|^{2} \quad \text { for } \quad \alpha \in \mathbb{C}
$$

where $g$ is any vector of unit norm in $\Gamma(\mathbb{C})$

Because of (20), it follows that

$$
p(\alpha, g) \geq 0, \quad \int_{\alpha \in \mathbb{C}} d \mu(\alpha) p(\alpha, g)=1
$$

hold for any vector $g$ of unit norm in $\Gamma(\mathbb{C})$.

\section{PHASE DISTRIBUTION}

To obtain phase distribution we consider first the phase operator

$$
P=\left(\frac{\lambda_{N+1}}{\lambda_{N}}-\frac{\lambda_{N}}{\lambda_{N-1}}+a^{*} a\right)^{-1 / 2} a
$$

and try to find the solution of the following eigenvalue equation

$$
P f_{\beta}=\beta f_{\beta}
$$

where $f_{\beta}=\Sigma a_{n}|n\rangle$.

Now,

$$
\begin{aligned}
P f_{\beta} & =\sum_{n=0}^{\infty} a_{n}\left(\frac{\lambda_{N+1}}{\lambda_{N}}-\frac{\lambda_{N}}{\lambda_{N-1}}+a^{*} a\right)^{-1 / 2} a|n\rangle \\
& =\sum_{n=1}^{\infty} a_{n}\left(\frac{\lambda_{N+1}}{\lambda_{N}}-\frac{\lambda_{N}}{\lambda_{N-1}}+a^{*} a\right)^{-1 / 2} \frac{\lambda_{n}}{\lambda_{n-1}}|n-1\rangle \\
& =\sum_{n=1}^{\infty} a_{n} \cdot \frac{\lambda_{n}}{\lambda_{n-1}}\left(\frac{\lambda_{N+1}}{\lambda_{N}}-\frac{\lambda_{N}}{\lambda_{N-1}}+a^{*} a\right)^{-1 / 2}|n-1\rangle \\
& =\sum_{n=1}^{\infty} a_{n} \cdot \frac{\lambda_{n}}{\lambda_{n-1}}\left(\frac{\lambda_{n}}{\lambda_{n-1}}-\frac{\lambda_{n-1}}{\lambda_{n-2}}+\frac{\lambda_{n-1}}{\lambda_{n-2}}\right)^{-1 / 2}|n-1\rangle \\
& =\sum_{n=0}^{\infty} a_{n+1} \cdot \frac{\lambda_{n+1}}{\lambda_{n}}\left(\frac{\lambda_{n+1}}{\lambda_{n}}\right)^{-1 / 2}|n\rangle \\
& =\sum_{n=0}^{\infty} a_{n+1} \cdot\left(\frac{\lambda_{n+1}}{\lambda_{n}}\right)^{1 / 2}|n\rangle
\end{aligned}
$$




$$
\beta f_{\beta}=\sum_{n=0}^{\infty} \beta a_{n}|n\rangle .
$$

From (24)-(26) we see that $a_{n}$ satisfies the following difference equation:

$$
a_{n+1}\left(\frac{\lambda_{n+1}}{\lambda_{n}}\right)^{1 / 2}=\beta a_{n}
$$

That is

$$
a_{n+1}=\beta\left(\frac{\lambda_{n+1}}{\lambda_{n}}\right)^{-1 / 2} a_{n}
$$

Hence,

$$
\begin{aligned}
& a_{1}=\beta\left(\frac{\lambda_{1}}{\lambda_{0}}\right)^{-1 / 2} a_{0} . \\
& a_{2}=\beta\left(\frac{\lambda_{2}}{\lambda_{1}}\right)^{-1 / 2} a_{1}=\beta^{2}\left(\frac{\lambda_{2}}{\lambda_{1}}\right)^{-1 / 2}\left(\frac{\lambda_{1}}{\lambda_{0}}\right)^{-1 / 2} a_{0}=\beta^{2}\left(\frac{\lambda_{2}}{\lambda_{0}}\right)^{-1 / 2} a_{0} . \\
& a_{3}=\beta\left(\frac{\lambda_{3}}{\lambda_{2}}\right)^{-1 / 2} a_{2}=\beta^{3}\left(\frac{\lambda_{3}}{\lambda_{2}}\right)^{-1 / 2}\left(\frac{\lambda_{2}}{\lambda_{0}}\right)^{-1 / 2} a_{0}=\beta^{3}\left(\frac{\lambda_{3}}{\lambda_{0}}\right)^{-1 / 2} a_{0}
\end{aligned}
$$

and so on.

Thus,

$$
a_{n}=\beta^{n} \cdot\left(\frac{\lambda_{n}}{\lambda_{0}}\right)^{-1 / 2} a_{0}=\beta^{n} \cdot\left(\lambda_{n}\right)^{-1 / 2} a_{0}
$$

Hence

$$
f_{\beta}=\sum_{n=0}^{\infty} a_{n}|n\rangle=a_{0} \sum_{n=0}^{\infty} \beta^{n} \cdot\left(\lambda_{n}\right)^{-1 / 2}|n\rangle .
$$

We take $a_{0}=1$ and $\beta=|\beta| e^{i \theta}$.

Then

$$
f_{\beta}=\sum_{n=0}^{\infty} e^{i n \theta}\left(\lambda_{n}\right)^{-1 / 2}|\beta|^{n}|n\rangle .
$$

Henceforth, we shall denote this vector as

$$
f_{\theta}=\sum_{n=0}^{\infty} e^{i n \theta}\left(\lambda_{n}\right)^{-1 / 2}|\beta|^{n}|n\rangle .
$$

where $0 \leq \theta \leq 2 \pi$ and call $f_{\theta}$ a phase vector in $\Gamma(\mathbb{C})$. 
Norm of the phase vector is given by

$$
\begin{aligned}
\left\|f_{\theta}\right\|^{2} & =\sum_{m, n=0}^{\infty} e^{i n \theta} e^{i m \theta}\left(\lambda_{n}\right)^{-1 / 2}\left(\lambda_{m}\right)^{1 / 2}|\beta|^{n+m}\langle n \mid m\rangle \\
& =\sum_{n=0}^{\infty} \frac{1}{\lambda_{n}}|\beta|^{2 n} \lambda_{n} \\
& =\sum_{n=0}^{\infty}|\beta|^{2 n} \\
& <\infty
\end{aligned}
$$

(if $|\beta|<1$ ).

The phase vectors are complete. We can show that

$$
I=\frac{1}{2 \pi} \int_{X} \int_{0}^{2 \pi} d \nu(x, \theta)\left|f_{\theta}\right\rangle\left\langle f_{\theta}\right|
$$

where

$$
d \nu(x, \theta)=d \mu(x) d \theta .
$$

Here we consider the set $X$ consisting of the points $x=0,1,2, \ldots$ and $\mu(x)$ is the measure on $X$ which equals

$$
\mu_{n}=\frac{1}{|\beta|^{2 n}}
$$

at the point $x=n$ and $\theta$ is the Lebesgue measure on the circle.

Define the operator

$$
\left|f_{\theta}\right\rangle\left\langle f_{\theta}\right|: \Gamma(\mathbb{C}) \rightarrow \Gamma(\mathbb{C})
$$

by

$$
\left|f_{\theta}\right\rangle\left\langle f_{\theta}\right| f=\left(f_{\theta}, f\right) f_{\theta}
$$

with $f=\sum_{n=0}^{\infty} a_{n}|n\rangle$

Now,

$$
\left(f_{\theta}, f\right)=\sum_{n=0}^{\infty} e^{-i n \theta}\left(\lambda_{n}\right)^{-1 / 2}|\beta|^{n} a_{n}
$$

and

$$
\left(f_{\theta}, f\right) f_{\theta}=\sum_{m, n=0}^{\infty} e^{i(m-n) \theta}\left(\lambda_{m}\right)^{-1 / 2}|\beta|^{m}\left(\lambda_{n}\right)^{1 / 2}|\beta|^{n} a_{n}|m\rangle
$$


Hence

$$
\begin{aligned}
\frac{1}{2 \pi} \int_{X} \int_{0}^{2 \pi} d \nu(x, \theta)\left|f_{\theta}\right\rangle\left\langle f_{\theta}\right| f= & \int_{X} d \mu(x) \sum_{m, n}\left(\lambda_{m}\right)^{-1 / 2}|\beta|^{m}\left(\lambda_{n}\right)^{1 / 2}|\beta|^{n} a_{n}|m\rangle \\
& \times \frac{1}{2 \pi} \int_{0}^{2 \pi} e^{-i(m-n) \theta} d \theta \\
= & \int_{X} d \mu(x) \sum_{n=0}^{\infty}|\beta|^{2 n}|n\rangle \\
= & \sum_{n=0}^{\infty} a_{n}|n\rangle|\beta|^{2 n} \frac{1}{|\beta|^{2 n}} \\
= & \sum_{n=0}^{\infty} a_{n}|n\rangle \\
= & f .
\end{aligned}
$$

We use the vectors $f_{\theta}$ to associate, to a given density operator $\rho$, a phase distribution as follows:

$$
\begin{aligned}
P(\theta) & =\frac{1}{2 \pi}\left(f_{\theta}, \rho f_{\theta}\right) \\
& =\frac{1}{2 \pi} \sum_{m, n=0}^{\infty}|\beta|^{m} \cdot|\beta|^{n} \cdot e^{i(n-m)} \cdot\left(\frac{|m\rangle}{\sqrt{\lambda_{m}}}, \rho \frac{|n\rangle}{\sqrt{\lambda_{n}}}\right) .
\end{aligned}
$$

The $P(\theta)$ as defined in (32) is positive, owing to the positivity of $\rho$, and is normalized

$$
\int_{X} \int_{0}^{2 \pi} P(\theta) d \nu(x, \theta)=1
$$

where

$$
d \nu(x, \theta)=d \mu(x) d \theta \text {. }
$$

for,

$$
\begin{aligned}
\int_{X} \int_{0}^{2 \pi} P(\theta) d v(x, \theta) & =\int_{X} d \mu(x) \sum_{m, n=0}^{\infty}|\beta|^{m} \cdot|\beta|^{n} \cdot \frac{1}{2 \pi} e^{i(n-m)} d \theta \cdot\left(\frac{|m\rangle}{\sqrt{\lambda_{m}}}, \rho \frac{|n\rangle}{\sqrt{\lambda_{n}}}\right) \\
& =\int_{X} d \mu(x) \sum_{n=0}^{\infty}|\beta|^{2 n} \cdot\left(\frac{|n\rangle}{\sqrt{\lambda_{n}}}, \rho \frac{|n\rangle}{\sqrt{\lambda_{n}}}\right) \\
& =\sum_{n=0}^{\infty}\left(\frac{|n\rangle}{\sqrt{\lambda_{n}}}, \rho \frac{|n\rangle}{\sqrt{\lambda_{n}}}\right) \\
& =1
\end{aligned}
$$


The phase distribution over the window $0 \leq \theta \leq 2 \pi$ for any vector $f$ is then defined by

$$
P(\theta)=\frac{1}{2 \pi}\left|\left(f_{\theta}, f\right)\right|^{2}
$$

\section{PHASE MEASUREMENT}

Once we have the POM information we are ready to discuss the phase estimation problem. Without loss of generality, we assume that $0 \leq \theta \leq 2 \pi$. The class of POMs we must optimize over in order to find the best phase estimate is taken to be

$$
\{d \hat{\Pi}(\theta): 0 \leq \theta \leq 2 \pi\},
$$

where

$$
d \hat{\Pi}(\theta)=d[\hat{\Pi}(\theta)]^{\dagger} \quad \text { and } \quad I=\int_{0}^{2 \pi} d \hat{\Pi}(\theta) .
$$

The conditional probability density, given the phase operator

$$
P=\left(\frac{\lambda_{N+1}}{\lambda_{N}}-\frac{\lambda_{N}}{\lambda_{N-1}}+a^{*} a\right)^{-1 / 2} a
$$

for obtaining a phase value $\theta$ from this POM is

$$
p(\theta, P)=\frac{(g, d \hat{\Pi}(\theta) g)}{d v(x, \theta)}, \quad \text { for } \quad 0 \leq \theta \leq 2 \pi, x \text { an integer }
$$

where $g$ is a vector of unit norm in $\Gamma(\mathbb{C})$.

We choose the POM, $d \hat{\Pi}(\theta)$, and the input vector $g$ to optimize our estimate of the phase shift $P$. For a given POM and the input vector, Eq. (37) supplies the PDF needed to perform classical maximal likelihood estimation. The observed phase value $\theta$ is our estimate of $P$. In order for this estimate to be one of maximum likelihood, we restrict our attention to the POMs satisfying

$$
P_{M L}(\theta)=\arg \max _{\theta} p(\theta, P), \quad \text { for } \quad 0 \leq \theta \leq 2 \pi
$$

and optimize our estimate over $d \hat{\Pi}$ and $g$ by maximizing the peak likelihoodminimizing $\delta \theta \equiv \frac{1}{p(\theta, P)}$.

For the input vector

$$
g=\sum_{n=0}^{\infty}\left(f_{n}, g\right) f_{n}
$$

where

$$
\left(f_{n}, g\right)=\left|\left(f_{n}, g\right)\right| e^{i k_{n}}, \quad n=0,1,2, \ldots,
$$


$\delta \theta$ is minimized by the following POM

$$
d \hat{\Pi}(\theta)=\left|f_{\theta}^{g}\right\rangle\left\langle f_{\theta}^{g}\right| d \nu(x, \theta)
$$

where

$$
d \nu(x, \theta)=d \mu(x) d \theta, \quad 0 \leq \theta \leq 2 \pi
$$

as in (34) and

$$
f_{\theta}^{g} \equiv \sum_{n=0}^{\infty} e^{i n \theta+i k_{n}}\left(\lambda_{n}\right)^{1 / 2}|\beta|^{n} f_{n} .
$$

To calculate reciprocal peak likelihood $\delta \theta$ with this optimum POM to estimate $P$ we observe first

$$
\begin{aligned}
p(\theta, P) & =\frac{(g, d \hat{\Pi}(\theta) g)}{d v(x, \theta)} \\
& =\left|\left(f_{\theta}^{g}, g\right)\right|^{2} \\
& =\left.\left.\left|\sum_{n=0}^{\infty} e^{-i n \theta-i k_{n}}\left(\lambda_{n}\right)^{1 / 2}\right| \beta\right|^{n}\left(f_{n}, g\right)\right|^{2} \\
& =\left.\left.\left|\sum_{n=0}^{\infty} e^{-i n \theta}\left(\lambda_{n}\right)^{1 / 2}\right| \beta\right|^{n}\left|\left(f_{n}, g\right)\right|\right|^{2}
\end{aligned}
$$

Hence a suitable peak likelihood $\delta \theta$ for maximum $p(\theta, P)$ can be (Helstrom, 1976),

$$
\begin{aligned}
\delta \theta & =\left|\left(f_{\theta}^{g}, g\right)\right|^{-2} \\
& =\left.\left.\left|\sum_{n=0}^{\infty}\left(\lambda_{n}\right)^{1 / 2}\right| \beta\right|^{n}\left|\left(f_{n}, g\right)\right|\right|^{-2}
\end{aligned}
$$

which is independent of the phases $\left\{k_{n}\right\}$. In fact, $p(\theta, P)$ is independent of the phases $\left\{k_{n}\right\}$.

As peak likelihood $\delta \theta$ is independent of $\left\{k_{n}\right\}$ we can assume, without loss of generality, that the input vector $g=\sum_{n=0}^{\infty}\left(f_{n}, g\right) f_{n}$ has positive real coefficient $\left(f_{n}, g\right)$. Equation (41) then reduces to

$$
f_{\theta}^{g}=f_{\theta}=\sum_{n=0}^{\infty} e^{i n \theta} \cdot\left(\lambda_{n}\right)^{1 / 2}|\beta|^{n} f_{n}
$$

for $0 \leq \theta \leq 2 \pi$ which is the solution of the eigenvalue equation (24)

$$
P f_{\theta}=e^{i \theta} f_{\theta} .
$$


Now we consider the operator

$$
U=\sum_{n=0}^{\infty} e^{-i k_{n}}\left|f_{n}\right\rangle\left\langle f_{n}\right| .
$$

Observe that

$$
U U^{*}=U^{*} U=I \text {. }
$$

Thus, $U$ is a unitary transformation.

Now, for an arbitrary input vector, $g$, the optimum POM from Eq. (40) is equivalent to performing the unitary transformation $U$ followed by the POM

$$
d \Pi(\theta)=\left|f_{\theta}\right\rangle\left\langle f_{\theta}\right| d \nu(x, \theta)
$$

where

$$
d \nu(x, \theta)=d \mu(x) d \theta, 0 \leq \theta \leq 2 \pi
$$

as in (27) and (28) for,

$$
\begin{aligned}
U f_{\theta}^{g} & =\sum_{n=0}^{\infty} e^{i n \theta+i k_{n}} \cdot e^{-i k_{n}}\left(\lambda_{n}\right)^{1 / 2}|\beta|^{n} f_{n} \\
& =\sum_{n=0}^{\infty} e^{i n \theta} \cdot\left(\lambda_{n}\right)^{1 / 2}|\beta|^{n} f_{n} \\
& =f_{\theta}
\end{aligned}
$$

where $f_{\theta}^{g}$ is given by (41).

Shifting the input vector's phase by the phase operator $P$ amounts to

$$
\left(f_{n}, g\right) \rightarrow e^{i n \theta_{0}}\left(f_{n}, g\right) \text { for } n=0,1,2, \ldots
$$

By rotating out the input phases $k_{n}$ with the $U$ transformation we get the transformed input as

$$
e^{i n \theta_{0}}\left(f_{n}, g\right) \stackrel{U}{\rightarrow} e^{i n \theta_{0}}\left|\left(f_{n}, g\right)\right|
$$

The effect of POM in Eq. (46) to this transformed vector

$$
g^{\prime}=\sum_{n=0}^{\infty} e^{i n \theta_{0}}\left|\left(f_{n}, g\right)\right| f_{n}
$$

gives the classical phase with PDF

$$
\begin{aligned}
p(\theta, P) & =\frac{\left(g^{\prime}, d \Pi(\theta) g^{\prime}\right)}{d v(x, \theta)} \\
& =\left(g^{\prime},\left|f_{\theta}\right\rangle\left\langle f_{\theta}\right| g^{\prime}\right)
\end{aligned}
$$




$$
\begin{aligned}
& =\left|\left(f_{\theta}, g^{\prime}\right)\right|^{2} \\
& =\left.\left.\left|\sum_{n=0}^{\infty} e^{i n\left(\theta_{0}-\theta\right)}\left(\lambda_{n}\right)^{1 / 2}\right| \beta\right|^{n}\left|\left(f_{n}, g\right)\right|\right|^{2}
\end{aligned}
$$

From the above equation it is clear that ML estimate obeys $P_{M L}(\theta)=\theta$.

Thus, the POM in Eq. (27) leads to the ML phase estimate for all vectors in $\Gamma(\mathbb{C})$. Thus, to achieve our goal of jointly optimizing phase estimate performance over both the measurement and the input vector, it remains for us to minimize $\delta \theta$ from Eq. (43), by appropriate choice of input vector. Specifically, the coefficients $\left\{\left(f_{n}, g\right)\right\}$ for the input vector must minimize the right side of the Eq. (43) subject to the normalization constraint

$$
\sum_{n=0}^{\infty}\left|\left(f_{n}, g\right)\right|^{2}=1
$$

and the average number constraint

$$
\sum_{n=0}^{\infty} \frac{\lambda_{n}}{\lambda_{n-1}}\left|\left(f_{n}, g\right)\right|^{2}=N_{0}
$$

where $N_{0}=\left(g, a^{*} a g\right)$.

Without loss of generality, we shall assume that $\left(f_{n}, g\right)$ are positive real.

Now, Maximize

$$
\begin{aligned}
L\left(g, \mu_{1}, \mu_{2}\right) \equiv & {\left[\sum_{n=0}^{\infty}\left(f_{n}, g\right)\right]^{2}+\mu_{1}\left[\sum_{n=0}^{\infty}\left(f_{n}, g\right)^{2}-1\right] } \\
& +\mu_{2}\left[\sum_{n=0}^{\infty} \frac{\lambda_{n}}{\lambda_{n-1}}\left(f_{n}, g\right)^{2}-N_{0}\right],
\end{aligned}
$$

where $\mu_{1}$ and $\mu_{2}$ are Lagrange's multipliers.

It is straight forward to show that

$$
\left(f_{n}, g\right)=\frac{c}{k+\frac{\lambda_{n}}{\lambda_{n-1}}} \quad \text { for } n=0,1,2, \ldots
$$

achieves the required stationary point for $L$, where $c$ and $k$ are positive constants depending on the Lagrange's multipliers. For brevity we shall chose $k=1$.

Now we choose $\left\{\lambda_{n}\right\}$ such that $\frac{\lambda_{n}}{\lambda_{n-1}} \geq n$. Then we have

$$
\frac{c /\left(1+\frac{\lambda_{n}}{\lambda_{n-1}}\right)}{1 / n} \leq \frac{c}{1 / n+1}
$$


Hence we see that

$$
\lim _{n \rightarrow \infty} \frac{c /\left(1+\frac{\lambda_{n}}{\lambda_{n-1}}\right)}{1 / n} \leq c .
$$

Thus, the series $\sum_{n=0}^{\infty} \frac{c}{1+\frac{\lambda_{n}}{\lambda_{n-1}}}$ and $\sum_{n=0}^{\infty} \frac{1}{n}$ converge or diverge together. But $\sum_{n=0}^{\infty} \frac{1}{n}$ diverges. Hence, we must introduce a truncation parameter in Eq. (55). That is, we have

$$
\begin{aligned}
\left(f_{n}, g\right) & =\frac{c}{1+\frac{\lambda_{n}}{\lambda_{n-1}}} \text { for } n=0,1,2, \ldots s \\
& =0 \text { for } n>s
\end{aligned}
$$

Now, we have

$$
\begin{aligned}
N_{0} & =\sum_{n=0}^{s} \frac{\lambda n}{\lambda n-1} \cdot\left|\left(f_{n}, g(\alpha)\right)\right|^{2} \\
& =\sum_{n=0}^{s} \frac{\lambda n}{\lambda n-1} \cdot \frac{c^{2}}{\left(1+\frac{\lambda_{n}}{\lambda_{n-1}}\right)^{2}} \\
& =\sum_{n=0}^{s} \frac{c^{2}}{1+\frac{\lambda_{n}}{\lambda_{n-1}}}-1
\end{aligned}
$$

where we have used Eqs. (52), (53), (54), and (56) with the truncation point $s$.

Then,

$$
\begin{aligned}
\delta \theta & =\left.\left.\left|\sum_{n=0}^{s}\left(\lambda_{n}\right)^{1 / 2}\right| \beta\right|^{n}\left(f_{n}, g\right)\right|^{-2} \\
& =\left.\left.c^{2} \cdot\left|\sum_{n=0}^{s}\left(\lambda_{n}\right)^{1 / 2}\right| \beta\right|^{n} \frac{c^{2}}{1+\frac{\lambda_{n}}{\lambda_{n-1}}}\right|^{-2} \\
& =c^{2} A \cdot\left|\sum_{n=0}^{s} \frac{c^{2}}{1+\frac{\lambda_{n}}{\lambda_{n-1}}}\right|^{-2} \\
& =\frac{c^{2} A}{\left(N_{0}+1\right)^{2}} \\
& \approx \frac{c^{2} A}{\left(N_{0}^{2}\right)}
\end{aligned}
$$

for $N_{0} \gg 1$. Here $A$ is a constant. 


\section{CONCLUSION}

We know (Shapiro and Shepard, 1991) that ML phase estimation with optimized state leads to $\delta \theta \sim \frac{1}{N_{0}^{2}}$ reciprocal peak likelihood performance where we are interested in the behavior at high average photon number, namely $N_{0} \gg 1$. In this paper we show that in the interacting Fock space case $\delta \theta$ can even be less than $\frac{1}{N_{0}^{2}}$.

\section{REFERENCES}

Accardi, L. and Bozejko, M. (1998). Interacting Fock space and gaussianization of probability measures. Infinite Dimensional Analysis, Quantum Probability and Related Topics 1(4), 663-670.

Accardi, L. and Nhani, M. (2001). The interacting Fock space of Haldane's exclusion statistics. preprint. Agarwal, G. S., Chaturvedi, S., Tara, K., and Srinivasan, V. (1992). Classical phase changes in nonlinear processes and their quantum counterparts. Physical Review A 45, 4904.

Bondurant, R. S. and Shapiro, J. H. (1984). Squeezed states in phase-sensing interferometers. Physical Review D 30, 2548.

Carruthers, P. and Nieto, M. M. (1968). Phase and angle variables in quantum Mechanics. Reviews of Modern Physics 40, 411.

Das, P. K. (2002). Coherent states and squeezed states in interacting Fock space. International Journal of Theoretical Physics 41(6).

Dirac, P. A. M. (1927). Proceedings of the Royal Society of London, Series A 114, 243.

Helstrom, C. W. (1976). Quantum Detection and Estimation Theory, Academic Press, New York.

Pegg, D. T. and Barnett, S. M. (1989). Unitary phase operator in quantum mechanics. Europhysics Letters 6, 483.

Shapiro, J. H. and Shepard, S. R. (1991). Quantum phase measurement: A system-theory perspective. Physical Review A 43, 3795.

Shapiro, J. H., Shepard, S. R., and Wong, N. C. (1989). Ultimate quantum limits on phase measurement. Physical Review Letters 62, 2377.

Susskind, L. and Glogower, J. (1964). Physics 1, 49. 